Am. J. Trop. Med. Hyg., 102(2), 2020, pp. 403-407

doi:10.4269/ajtmh.19-0720

Copyright $(\odot) 2020$ by The American Society of Tropical Medicine and Hygiene

\title{
Heart Rate Variability as an Indicator of Autonomic Nervous System Disturbance in Tetanus
}

\begin{abstract}
Ha Thi Hai Duong, ${ }^{1}$ Girmaw Abebe Tadesse, ${ }^{2}$ Phung Tran Huy Nhat, ${ }^{3}$ Nguyen Van Hao, ${ }^{1,4}$ John Prince, ${ }^{2}$ Tran Duc Duong, ${ }^{1}$ Trịnh Trung Kien, ${ }^{3}$ Le Thanh Hoang Nhat, ${ }^{3}$ Le Van Tan, ${ }^{3}$ Chris Pugh, ${ }^{5}$ Huynh Thi Loan, ${ }^{1}$ Nguyen Van Vinh Chau, ${ }^{1}$ Lam Minh Yen, ${ }^{3}$ Tingting Zhu, ${ }^{2}$ David Clifton, ${ }^{2}$ and Louise Thwaites ${ }^{3,6 *}$

${ }^{1}$ Hospital for Tropical Diseases, Ho Chi Minh City, Vietnam; ${ }^{2}$ Institute of Biomedical Engineering, University of Oxford, Oxford, United Kingdom; ${ }^{3}$ Oxford University Clinical Research Unit, Ho Chi Minh City, Vietnam; ${ }^{4}$ University of Medicine and Pharmacy, Ho Chi Minh City, Vietnam; ${ }^{5}$ Nuffield Department of Medicine, University of Oxford, Oxford, United Kingdom; ${ }^{6}$ Centre for Tropical Medicine and Global Health, University of Oxford,
\end{abstract} Oxford, United Kingdom

\begin{abstract}
Autonomic nervous system dysfunction (ANSD) is a significant cause of mortality in tetanus. Currently, diagnosis relies on nonspecific clinical signs. Heart rate variability (HRV) may indicate underlying autonomic nervous system activity and represents a potentially valuable noninvasive tool for ANSD diagnosis in tetanus. HRV was measured from three 5-minute electrocardiogram recordings during a 24-hour period in a cohort of patients with severe tetanus, all receiving mechanical ventilation. HRV measurements from all subjects_five with ANSD (Ablett Grade 4) and four patients without ANSD (Ablett Grade 3)—showed HRV was lower than reported ranges for healthy individuals. Comparing different severities of tetanus, raw data for both time and frequency measurements of HRV were reduced in those with ANSD compared with those without. Differences were statistically significant in all except root mean square SD, indicating HRV may be a valuable tool in ANSD diagnosis.
\end{abstract}

Tetanus is a severe disease characterized by toxin-mediated disinhibition of autonomic and motor nervous systems. Motor neuron disinhibition causes characteristic muscle spasms, whereas autonomic nervous system disinhibition results in fluctuating blood pressure, tachycardia, and pyrexia. When mechanical ventilation is available, spasms can be controlled, but autonomic nervous system dysfunction (ANSD) remains a principal cause of mortality. ${ }^{1,2}$ Robust methods of detecting ANSD suitable for implementation in resource-limited settings where most tetanus occurs would allow earlier intervention and may improve outcome. Diagnosis is currently based on nonspecific clinical signs of pyrexia, sweating, and increased or fluctuating heart rate and blood pressure. ${ }^{3}$ Other methods include 24-hour collections of urinary catecholamines, but this has low specificity, unsuitable for routine use. ${ }^{4}$

In health, heart rate is carefully controlled by the autonomic nervous system. Alterations in parasympathetic and sympathetic nervous system activity result in beat-to-beat heart rate variation, and hence, this variation (heart rate variability [HRV]) reflects autonomic nervous system activity. Heart rate variability is altered in pathological states, such as ischemic heart disease, and reduced variability is predictive of worse outcomes. ${ }^{5}$ Standardized measures of HRV can be calculated from electrocardiogram (ECG) R-R intervals, and consensus guidelines on appropriate indicators are available. ${ }^{5}$ Time domain variables are calculated directly from $R-R$ intervals (termed normal-to-normal intervals), for example, SD. Frequency domain variables are generated from ECG spectral analysis, usually following fast Fourier transformation. ${ }^{5}$ By observing changes in these components after administering autonomic nervous system antagonists, relative contributions of parasympathetic and sympathetic nervous systems have been inferred. Whereas total power of the spectrum represents the general level of autonomic activation, low-frequency activity $(<0.15 \mathrm{~Hz})$ is mainly due to baroreceptor reflex modulation and related to both vagal and sympathetic influence,

*Address correspondence to Louise Thwaites, Hospital for Tropical Diseases, 764 Vo Van Kiet, Quan 5, Ho Chi Minh City, Vietnam. E-mail: Ithwaites@oucru.org and high-frequency activity is mainly aligned with vagal activity. Low to high frequency ratio is accepted to indicate the balance between both systems; however, this interpretation fails to take account of effects such as different temporal patterns of sympathetic and parasympathetic components and cardiac pacemaker sensitivity.

Heart rate variability changes in tetanus are largely unknown. Sykora et al. ${ }^{6}$ analyzed baroreflex sensitivity and time domain variables in an 87-year-old woman with tetanus and reported decreased baroreceptor sensitivity compared with a control of similar age; however, the patient, but not control, received mechanical ventilation and a beta-blocker, both of which can influence sensitivity. Goto et al. ${ }^{7}$ reported reduced frequency domain variables in an 11-year-old child; however, this recording was taken following a cardiac arrest and on the 122nd day of hospitalization, when clinical recovery from tetanus is normally expected.

Nevertheless, ANSD diagnosis and prognostication through HRV remains an attractive prospect because of its noninvasive nature. Hitherto, required monitoring equipment was rarely available in settings where most tetanus occurs, but growing availability of low-cost sensors means measurement is increasingly feasible in low-resource settings. ${ }^{8}$ In this study, we aim to investigate the relationship of HRV and ANSD in patients with severe tetanus, providing proof-of-principle that such monitoring may be valuable.

The study was conducted in the Intensive Care Unit at the Hospital for Tropical Diseases, Ho Chi Minh City, between October 2016 and January 2017 and was approved by the Ethical Committee of the Hospital for Tropical Diseases. Written informed consent was given by all participants or representatives before enrollment.

Adults with severe tetanus (Ablett Grade 3 or 4) diagnosed according to the Hospital for Tropical Disease guidelines ${ }^{9,10}$ and receiving mechanical ventilation were recruited to the study. Recruitment was pragmatic and depended on availability of suitable monitors. Ablett Grade 3 was defined as "severe spasms interfering with breathing" and Grade 4 as Grade 3 but with ANSD. ${ }^{10}$ Autonomic nervous system dysfunction was diagnosed clinically by the attending physician 
but required the presence of at least three of the following within 12 hours: heart rate $>100 \mathrm{bpm}$, systolic blood pressure $>140 \mathrm{mmHg}$, blood pressure fluctuation with minimum mean arterial pressure $<60 \mathrm{mmHg}$, and temperature $>38^{\circ} \mathrm{C}$ without evidence of intercurrent infections.

Tetanus management followed a standard protocol previously described, ${ }^{11}$ consisting of antibiotics, and spasm control using benzodiazepines and pipecuronium. Autonomic nervous system dysfunction was managed principally with magnesium sulfate.

Electrocardiogram data were collected from bedside monitors (Datex; Datex Ohmeda Inc., GE Healthcare, Helsinki, Finland) in supine undisturbed patients using VSCapture software. ${ }^{12}$ Electrocardiogram, physiological, and clinical data were collected over a 24-hour period. Heart rate variability features were extracted from noise-free 5-minute recordings at 6 AM, 12 noon, and $6 \mathrm{PM}$ to prevent bias from HRV diurnal variation. ${ }^{13}$ Time domain variables measured were square root of the mean squared differences of successive normal-to-normal intervals (RMSSD) and SD of all normal-to-normal intervals (SDNN). Frequency domain variables were total power, highfrequency power $(0.15-0.4 \mathrm{~Hz})$, low-frequency power (0.05-0.15 Hz), low-frequency normalized units, highfrequency normalized units, and low- to high-frequency ratio. Statistical analyses were performed using $R$ statistical software version 3.5.1 (R Corporation, Vienna, Austria). Data are presented as mean (SD). Heart rate variability was compared between two groups of tetanus severity using a linear mixed-effects model to correct for repeated measurements. A $P$-value $<0.05$ was considered statistically significant.

Five patients with Ablett Grade 4 and 5 patients with Ablett Grade 3 tetanus were recruited to the study. Data from one patient with Grade 3 tetanus was too noisy for analysis and, therefore, excluded. Clinical characteristics of the remaining nine patients are given in Table 1. Of these, $8 / 9$ had three highquality noise-free 5-minute segments at the chosen time point. One patient with Ablett Grade 4 had only two suitable 5-minute segments at 12 noon and $6 \mathrm{PM}$.

Heart rate variability data are presented in Figure 1 and Table 2. All HRV measurements were very low compared with reported ranges for healthy individuals, with low- to highfrequency ratios being significantly greater. ${ }^{5}$ Comparing different severities of tetanus, both time (RMSSD and SDNN) and frequency (low frequency, high frequency, low-frequency normalized units, and total power) variables were reduced in those with ANSD (Ablett Grade 4) compared with those without. Differences were statistically significant in all except $\operatorname{RMSSD}(P=0.09)$. Only high-frequency normalized units and low- to high-frequency ratios showed no difference between groups.

We present, to our knowledge, the first HRV measurements in a series of patients with tetanus. Our data show a consistent reduction in time and frequency domain variables compared with values reported in healthy subjects. These are particularly reduced in those with clinical signs of ANSD. This is consistent with HRV reported in other pathological states with high levels of sympathetic activation and with existing understanding of ANSD in tetanus.

Sympathetic activation in tetanus is associated with increased circulating catecholamines, which are increased in proportion to disease severity. ${ }^{4}$ These may exert direct effects on the heart and vasculature and indirect effects through reflex reduction in vagal tone. The observed reduction in HRV variables in those with ANSD is consistent with sympathetic nervous system activation. Although the reduction in highfrequency power, suggesting a reduction in vagal tone, is expected, we also observed a reduction in low-frequency power, indicative of both sympathetic and parasympathetic

TABLE 1

Clinical data of patients

Ablett Grade $3,{ }^{\star} n=4$

$51.25(20.66)$

4 (100)

$5.8(6.5)$

$4(100)$

$0(0)$

$152.8(22.34)$

$59.00(6.21)$

$94.50(10.25)$

$127.60(2.27)$

$137.50(9.57)$

$115.00(5.77)$

$30.00(60)$

$120.00(80.80)$

$0(0)$

$38.40(7.83)$
Ablett Grade $4^{\star}$ (ANSD), $n=5$

$58(7.68)$

$4(80)$

4.8 (4.09)

5 (100)

$0(0)$

$210.2(29.26)$

48.20 (10.08)

$90.00(20.41)$

$159.90(21.06)$

$217.40(26.49)$

107.40 (27.24)

48.00 (65.72)

86.40 (84.17)

43.20 (26.29)

$30.72(4.29)$

the 24-hour study period (g)

Total dose of pipecuronium during the 24hour study period (mg) 


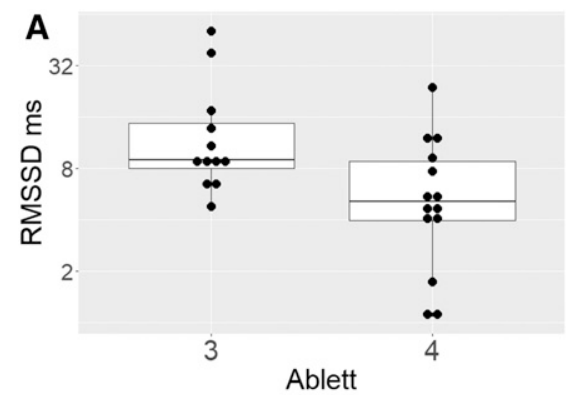

C

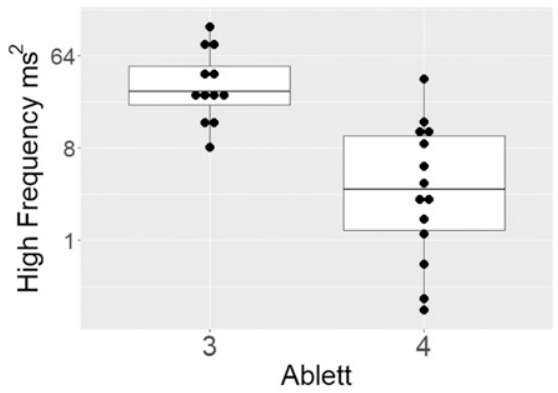

E

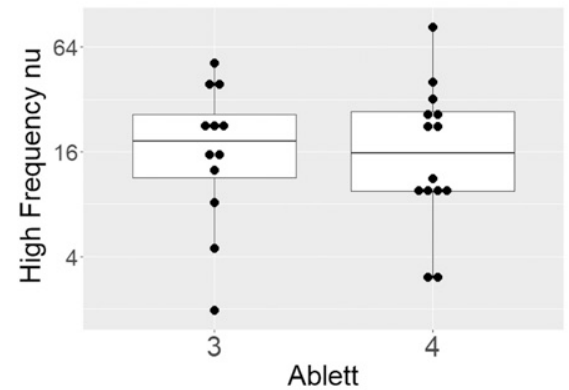

G

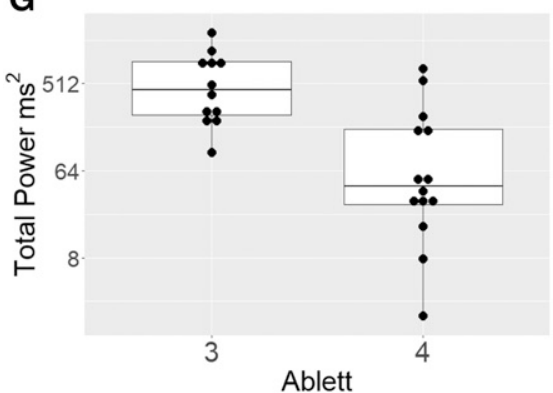

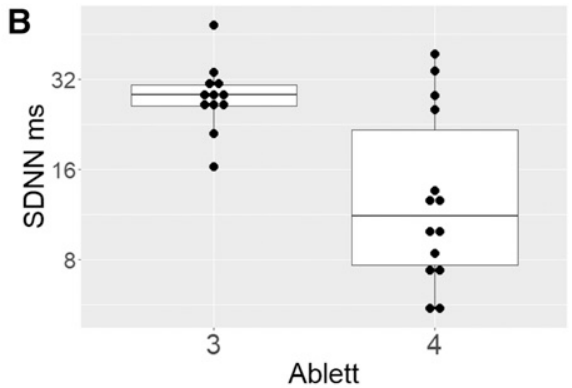

D

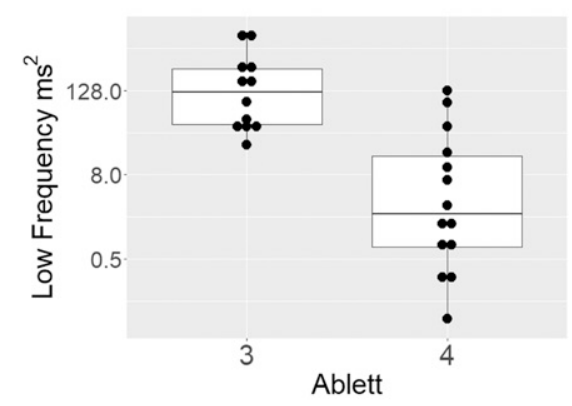

$\mathbf{F}$

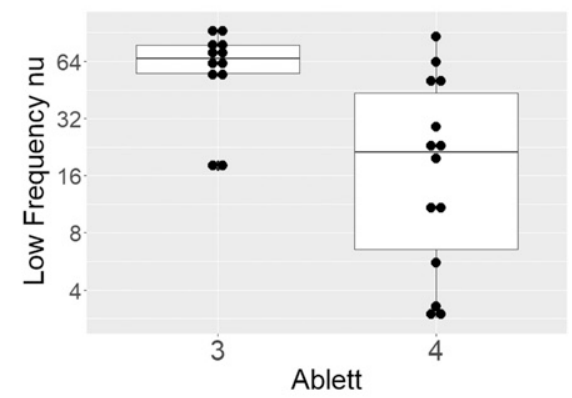

H

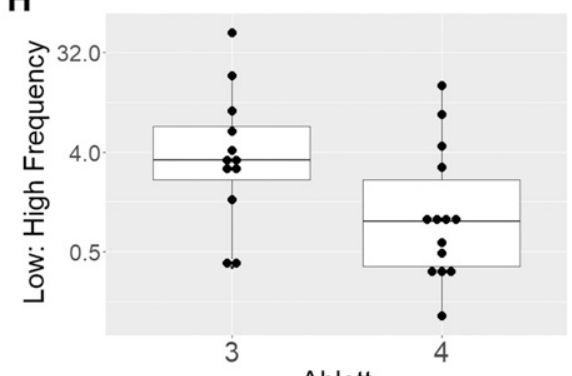

Ablett

FIGURE 1. Heart rate variability variables in patients according to Ablett Grade. (A) Square root of the mean squared differences of successive normal-normal (NN) intervals (RMSSD), (B) SD of all NN intervals (SDNN), (C) power in the high-frequency range, (D) power in the low-frequency range, (E) high-frequency power in normalized units, (F) low-frequency power in normalized units, $(\mathbf{G})$ total spectral power, and (H) low- to highfrequency ratio.

activation. In cases of sympathetic activation, heart rate increases and total power is reduced and, as a result, the lowfrequency component may actually decrease. ${ }^{14}$ Similarly at high levels of sympathetic stimulation, a "ceiling effect" may occur at the sinoatrial node when further response cannot occur. $^{14}$

A significant limitation to interpretation of our data is that our patients were all receiving sedative drugs which may influence $\mathrm{HRV}$. Although sedation is not reported to affect HRV in critically ill patients ${ }^{15}$ and subjects in both groups received similar sedative doses, it is possible that drugs were titrated against clinical effect. Magnesium sulfate was used almost exclusively in those with ANSD. Although we have previously shown its use in tetanus is associated with a reduction in urinary catecholamine excretion, ${ }^{16}$ limited data in myocardial infarction suggest that it has limited effect on HRV. ${ }^{17}$

A further limitation is that we used 5-minute recordings to measure time domain variables. Guidelines recommend that 
TABLE 2

Heart rate variability variables

\begin{tabular}{|c|c|c|c|}
\hline & Ablett Grade 3, $n=12$ & Ablett Grade 4 (ANSD), $n=14$ & $P$-value \\
\hline Root mean square SD (ms) & $8.94(8.03-14.71)$ & $5.13(3.95-8.86)$ & 0.09 \\
\hline SD of all normal-to-normal (ms) & $28.4(26.03-30.55)$ & $11.23(7.67-22.40)$ & 0.008 \\
\hline Low frequency $\left(\mathrm{ms}^{2}\right)$ & $127.94(42.37-262.09)$ & $2.29(0.76-14.95)$ & 0.006 \\
\hline High frequency $\left(\mathrm{ms}^{2}\right)$ & $28.80(21.65-52.81)$ & $3.17(1.28-10.54)$ & 0.004 \\
\hline Low frequency (normalized units) & $66.08(55.11-77.74)$ & $21.29(6.86-44.60)$ & 0.04 \\
\hline High frequency (normalized units) & $18.72(11.48-26.80)$ & $16.53(9.49-27.16)$ & 0.97 \\
\hline Total power $\left(\mathrm{ms}^{2}\right)$ & $447.4(243.3-867.6)$ & $44.46(28.28-172.22)$ & 0.01 \\
\hline Low- to high-frequency ratio & $3.41(2.32-7.01)$ & $0.94(0.37-2.44)$ & 0.17 \\
\hline
\end{tabular}

these should be measured from 24-hour recordings. ${ }^{5,14}$ Nevertheless, our values are lower than reported 5-minute "normal" values, and our primary comparison was between severity groups.

Heart rate control in tetanus is undoubtedly complex and influenced by many factors not measured in this study. As such, we aimed only to demonstrate that variability is related to disease severity and that alterations in HRV may aid ANSD diagnosis in patients with tetanus. Currently, ANSD diagnosis is limited by poor specificity and may be difficult to distinguish from other causes of cardiovascular instability, such as infection, ischemia, or pain. Heart rate variability could, therefore, potentially be a more sensitive and specific way of identifying those with ANSD. The low HRV observed even in patients with Grade 3 tetanus may represent a clinically less apparent category of ANSD, but where, nevertheless, intervention may be beneficial. Furthermore, HRV changes may be early predictors of subsequent ANSD and enable earlier intervention.

This article has focused on using established HRV measures; however, it is likely these are relatively blunt tools with which to decipher the complex underlying physiological mechanisms and rely on high-quality signals difficult to obtain in critically ill populations in resource-limited settings. However, it may be that obtaining high-quality data becomes more feasible through either increased availability of wearable devices or adaptation of existing equipment. ${ }^{18}$ As newer innovative methods for analyzing data are developed, for example, artificial intelligence, more sensitive ways of analysis could be developed, providing better insight into control mechanisms and disease pathophysiology.

Received September 28, 2019. Accepted for publication November 6, 2019.

Published online December 12, 2019.

Note: Supplemental table appears at www.ajtmh.org.

Acknowledgments: We thank all staff in the AICU at the Hospital for Tropical Diseases and the biostatistics group at the Oxford University Clinical Research Unit for statistical advice.

Financial support: This study was conducted with support from the Royal Academy of Engineering (FoRDF1718_3_19) and the Wellcome Trust (107367/Z/15/Z).

Disclosure: C. P. is a scientific cofounder of OxeHealth Ltd. C. P. reports other support from Oxehealth Ltd., outside the submitted work. In addition, C. P. has a patent Bayesian System for Phenotyping Patients using Biosignal Data pending to Oxford University Innovation Limited. N. V. H. has received travel support from Zuelling Pharma Vietnam.

Authors' addresses: Ha Thi Hai Duong, Nguyen Van Hao, Tran Duc Duong, Huynh Thi Loan, and Nguyen Van Vinh Chau, Hospital for Tropical Diseases, Ho Chi Minh City, Vietnam, E-mails: haduong200385@yahoo.com.vn, dr_ nguyenvanhao@ump.edu.vn, duongtran.bvbnd@gmail.com, loanhsuv@ gmail.com, and chaunv@@oucru.org. Girmaw Abebe Tadesse, John Prince, Tingting Zhu, and David Clifton, Institute of Biomedical Engineering, University of Oxford, Oxford, United Kingdom, E-mails: girmaw.abebe@eng.ox.ac.uk, john.prince.ox@gmail.com, tingting. zhu@eng.ox.ac.uk, and david.clifton@eng.ox.ac.uk. Phung Tran Huy Nhat, Trịnh Trung Kien, Le Thanh Hoang Nhat, Le Van Tan, Yen Lam Minh, and Louise Thwaites, Oxford University Clinical Research Unit, Ho Chi Minh City, Vietnam, E-mails: nhatpth@oucru.org, kientt@ oucru.org, nhatlth@oucru.org, tanlv@oucru.org, yenlm@oucru.org, and Ithwaites@oucru.org. Chris Pugh, Nuffield Department of Medicine, University of Oxford, Oxford, United Kingdom, E-mail: chris.pugh@ndm.ox.ac.uk.

This is an open-access article distributed under the terms of the Creative Commons Attribution (CC-BY) License, which permits unrestricted use, distribution, and reproduction in any medium, provided the original author and source are credited.

\section{REFERENCES}

1. Woldeamanuel YW, Andemeskel AT, Kyei K, Woldeamanuel MW, Woldeamanuel W, 2016. Case fatality of adult tetanus in Africa: systematic review and meta-analysis. $J$ Neurol Sci 368: 292-299.

2. Thwaites $C L$ et al., 2004. Impact of improved vaccination programme and intensive care facilities on incidence and outcome of tetanus in southern Vietnam, 1993-2002. Trans $R$ Soc Trop Med Hyg 98: 671-677.

3. Yen LM, Thwaites CL, 2019. Tetanus. Lancet 393: 1657-1668.

4. Thwaites CL, Yen LM, Cordon SM, Binh NT, Nga N, White NJ, Soni $\mathrm{N}$, MacDonald IA, Farrar JJ, 2006. Urinary catecholamine excretion in tetanus. Anaesthesia 61: 355-359.

5. Shaffer F, Ginsberg JP, 2017. An overview of heart rate variability metrics and norms. Front Public Heal 5: 1-17.

6. Sykora M, Diedler J, Veltkamp R, Steiner T, 2008. Autonomic impairment in tetanus: delayed baroreflex involvement. J Neuro/ Sci 270: 201-204.

7. Goto T, Fukushima H, Sasaki G, Matsuo N, Takahashi T, 2001. Evaluation of autonomic nervous system function with spectral analysis of heart rate variability in a case of tetanus. Brain Dev 23: 791-795.

8. Turner HC, Hao NV, Yacoub S, Hoang VMT, Clifton DA, Thwaites GE, Dondorp AM, Thwaites CL, Chau NVV, 2019. Achieving affordable critical care in income countries. BMJ Glob Heal 4: e001675.

9. Yen L, 2017. Huong Dan Chan Doan Va Dieu Tri Benh Vien Benh Nhiet Doi.

10. Ablett JJL, 1956. Tetanus and the anaesthetist: a review of the symptomatology and the recent advances in treatment. $\mathrm{Br} J$ Anaesth 28: 258-273.

11. Loan $\mathrm{H}$ et al., 2018. Intrathecal Immunoglobulin for treatment of adult patients with tetanus: a randomized controlled $2 \times 2$ factorial trial [version 1; referees: awaiting peer review]. Wellcome Open Res 3: 58.

12. Vital Signs Capture. Available at: https://sourceforge.net/p/ vscapture/blog/.

13. Pichot V, Roche F, Celle S, Barthélémy JC, Chouchou F, 2016. HRV analysis: a free software for analyzing cardiac autonomic activity. Front Physiol 7: 1-15. 
14. Task Force of the European Society of Caridiology and the North American Society of Pacing Electrophysiology, 1996. Hear rate variability: standards of measurement, physiological interpretation and clinical use. Circulation 93: 1043-1065.

15. Schmidt $\mathrm{H}$ et al., 2005. Autonomic dysfunction predicts mortality in patients with multiple organ dysfunction syndrome of different age groups. Crit Care Med 33: 1994-2002.

16. Thwaites CL, Yen LM, Cordon SM, Thwaites GE, Loan HT, Thuy TT, White NJ, Soni N, Macdonald IA, Farrar JJ, 2008. Effect of magnesium sulphate on urinary catecholamine excretion in severe tetanus. Anaesthesia 63: 719-725.
17. Parikka H, Toivonen L, Naukkarinen V, Tierala I, PohjolaSintonen S, Heikkilä J, Nieminen MS, 1999. Decreases by magnesium of QT dispersion and ventricular arrhythmias in patients with acute myocardial infarction. Eur Heart $J$ 20: 111-120.

18. Brandle J, PreissI H, Draganova R, Ortiz E, Kagan KO, Abele $\mathrm{H}$, Brucker SY, Kiefer-Schmidt I, 2015. Heart rate variability parameters and fetal movement complement fetal behavioral states detection via magnetography to monitor neurovegetative development. Front Hum Neurosci 9: $1-8$. 